to Hubble (Astrophysical Journal, 64, p. 321 ; 1926), is about $10^{81}$. Then, as Sir James Jeans (NATure, 121$, p. $674 ; 1928)$ points out, the probability of a universe with as little entropy as it possesses at present is of the order of $\left(10^{-10^{11 \cdot 6}}\right)^{10^{81}}$, or $10^{-10^{92.6}}$, assuming that an appreciable fraction of the present atoms are non-permanent, and that most of the energy of the present universe is in the form of radiation of frequency too small to build atoms readily. This probability is very small, but finite, and remains finite however large the finite universe may be. Now in the course of eternity any event with a finite probability will occur. Hence if the present universe melts away into radiation, another equally improbable will develop in the course of about $10^{10^{100}}$ years. But the improbabilities involved are so vast that it is perhaps unlikely that even a single abom will be built up from radiation in inter-galactic space during the 'life' of the present universe. Fluctuation can generally be neglected in practical life, but not $i_{x i}$ the contemplation of eternity.

If the above argument is correct, there is no need to assume a break in the order of Nature to account for the beginning of the present universe. In this case the time taken by such events as the "life' of a star is instantaneous in comparison with its re-creation; and eternity is, on the whole, dull. Indeed, all but $10^{-10^{100}}$ of it is duller than the present moment. But during most of eternity there can be no living creatures at all resembling ourselves to be bored. For since all organisms live by the utilisation of processes involving increase of entropy, they can presumably only exist during the aftermath of a very large fluctuation. This is why we are witnesses of this excessively unusual occurrence.

This letter is not intended to suggest that the above view of the universe is correct, but that it is consistent with Sir James Jeans's premisses, even if the actual numbers involved are very different from those here assumed.

J. B. S. HaLdane.

Sir William Dunn Institute,

University, Cambridge.

MAY a self-constituted Anubis of the scientific philosophy of 'the ancients' raise a plaint outside the door of the Temple of Modern Knowledge from which he hears the voice of its Secretary stating his opinion about their views? (Supp. NATURE, Nov. 3). The irreversibility of living energies was fundamental knowledge on which the ancient scientists of Aryavarta based their doctrines of evolution, human and cosmic ; and the ancients will be pleased to know that the moderns have, in their turn, discovered this law of Nature. Everything in the universe, from atoms to stars, is subject to 'birth ' (manifestation) and 'death' (withdrawal into latency). Periodical cycles of appearance and disappearance of all forms of life is the method of the evolution of consciousness. The phœnix rises out of the dead ashes at the close of the dark half (Pralaya) of the cycle. The ancients would agree with the view expressed in the notes on p. 703 referring to Sir James Jeans' lecture " that a degradation of the physical universe is not necessarily a degradation of the world of spirit." To them a Manvantara is a cycle of a solar system : a Mahamanvantara that of the cosmos. These cycles are "The days and nights of Brahmâ." Oxford has published "The Sacred Books of the East" in many volumes, but Cambridge is advised to read the English translations of Indian scholars, who understand better the technical language and scientific symbols of their ancestors.

W. W. L.

\section{An Attempt to Polarise Electron Waves by} Reflection.

An experiment has been made to test whether or not electron waves are polarised by reflection from the face of a crystal.

The experiment is similar in method to the double mirror experiment by which one demonstrates the polarisation of light by reflection from glass. A homogeneous beam of electrons is reflected at $45^{\circ}$ incidence from a $\{111\}$-face of a nickel crystal, and the reflected beam proceeding from the first crystal is then reflected at the same angle of incidence from a second similar crystal. A double Faraday box is placed to receive electrons proceeding from the second crystal in the direction of regular reflection, but only such electrons are allowed to enter the collector as have retained all or nearly all of their initial energy through the two reflections. Electrons which have lost amounts of energy corresponding to more than two equivalent volts are excluded by an opposing potential.

The second crystal and the collector are joined rigidly together, and the system comprising these parts may be rotated about an axis which coincides with the axis of the beam proceeding from the first to the second crystal. It is possible, therefore, to vary the dihedral angle between the plane of incidence of the second reflection and that of the first. There are two positions of the moveable system for which these planes coincide, and for these ' parallel' positions the current entering the collector should be a maximum provided asymmetry is impressed upon the electron beam at reflection ; for the intermediate 'transverse' positions the current should be a minimum.

The observation is that, if such a doubly periodic variation of the current with angle exists, its amplitude is less than two one-hundredths of the total current, which corresponds to the uncertainty of the measurements. So far as our observations go, there is no polarisation of electron waves by reflection.

The measurements have been made at bombarding potentials in the range 10 to 150 volts. Within this range the intensity of the doubly reflected beam exhibits five maxima, occurring at bombarding potentials $20,55,77,103$, and 120 volts. The maxima corresponding to the highest three of these voltages were observed in our earlier experiments on electron reflection (Proc. Nat. Acad. Sci., 14, 624 (Fig. 3); 1928). The two at the lower voltages were outside the range of these previous observations.

The beam at 120 volts is particularly strong, and with regard to this beam it may be stated that the amplitude of the variation due to polarisation is less than one-hundredth of the total current.

The current incident upon the first crystal is about $2 \times 10^{-4}$ amp., and the current received in the collector is of the order $5 \times 10^{-12} \mathrm{amp}$. C. J. Davisson.

Bell Telephone Laboratories, Inc. L. H. Germer. New York, N.Y., Oct. 19

\section{Radiovision.}

IN steering clear of the hybrid 'television' in a note in NaturE of Nov. 3, p. 704, you lead to a confusion of terms which, unfortunately, is common.

'Radiovision' is suitable for 'television' by radio, but 'television' can also be transmitted by telegraph or telephone line.

Near the end of the note you refer to "the radio pictures," meaning 'television.' The broadcast of radio pictures-phototelegraphy-is now being done by the B.B.C., and is in a state much nearer practicability than 'television.' 